\title{
MIMO-OFDM with Enhanced Channel Estimation based on DFT Interpolation
}

\author{
Mahdi Abdul Hadi \\ Asst. Professor \\ University of Baghdad \\ Electronic and Communication Engineering \\ Department
}

\author{
Omar Almukhtar Tawfeeq \\ MSc. Student \\ University of Baghdad \\ Electronic and Communication Engineering \\ Department
}

\begin{abstract}
In practical wireless communication systems, the transmitter send the information out through a multipath fading channel. At the receiver side, the received information distorted by the channel characteristic (due to multipath fading). So, in order to recover the transmitted information accurately, the channel effect must be known at the receiver. In this paper channel estimation for STBC-MIMO-OFDM system has been investigated by implementing the most popular channel estimators least Square (LS) and Minimum Mean Error Square (MMSE) both based on comb type pilot arrangement to estimate the channel effect at pilot locations, and channel interpolation between pilot locations was done using linear interpolation. The performance of LS and MMSE estimators can be improved by using DFT-based estimation technique. LS and MMSE estimator have been implemented with and without DFT-based estimation technique to estimate the channel effect for STBC-MIMO-OFDM system and compare their performance by measuring the bit error rate BER with QAM as modulation scheme and for different MIMO antenna configurations, also the channel between each antenna pair is assumed to be multipath Rayleigh Slow fading channel based on Clark's model.
\end{abstract}

\section{General Terms}

Channel estimation, communication, DFT algorithms.

\section{Keywords}

MIMO-OFDM, MMSE, Inter-symbol-Interference

(ISI), Channel estimation, DFT interpolation.

\section{INTRODUCTION}

In our present day, wireless communication applications such as internet browsing, video calling, and high definition video broadcast require high transmission rate, reliable communication system, and efficient transmission technique. High data rate transmission over wireless channel (frequency selective fading) is subject to Inter Symbol Interference (ISI) which increase the error rate that leads to low Quality of Service (QoS). Orthogonal Frequency Division Multiplexing (OFDM), is one of the most popular transmission technique that's known for its ability to reduce the effect of (ISI) by transforming the frequency selective fading channel into a set of parallel flat fading channel [1]. OFDM can be combined with Multiple Input Multiple Output (MIMO) antenna configuration based on the idea that the probability of multiple independent wireless fading channels that are in deep fading at the same time is very low [2]. Multiple antenna techniques can be divided into two types: diversity techniques and spatial-multiplexing techniques. The diversity techniques intend to transmitlreceive the same data signals over multiple antennas, and apply various transmission, detection, and combining techniques to improve the transmission reliability [3]. On the other hand, spatial-multiplexing techniques intend to transmitlreceive, independent data signals over multiple transmit antennas, to achieve high transmission speed [4]. So MIMO-OFDM combination is one of the most promising candidate for the $4 \mathrm{G}$ communications systems, but one of the most critical issues that significantly affect in the performance of the MIMO-OFDM and any other wireless communication system is the wireless fading channel especially in deep fading case [5]. So the scope of this paper is to implement the LS and MMSE channel estimators and enhance their performance by using DFT estimation technique.

The reminder of this paper is organized as follows. After describing the system model in details in Section 2, LS and MMSE channel estimation using comb-type pilot is described in Section 3 and the estimation enhancement based on DFT interpolation presented in Section 4. The simulation results are given in Section 5.

\section{SYSTEM MODEL}

Figure 1 , is a detailed block diagram for the $2 \times 2$ MIMOOFDM transceiver with improved DFT channel estimation. At the transmitter side, first the input binary data mapped according to the type and the order (M-PSK/M-QAM) of the signal mapper.

After that, a pilot data inserted along with the mapped data. Pilot insertion can be performed in two approaches, either by inserting pilot tones into all subcarriers of the OFDM symbols with a specific period or inserting pilot tones into each OFDM symbol at periodically-located subcarriers.

The first one, called block type pilot insertion, has been developed under the assumption of fast varying channel within one OFDM symbol. The second one, the comb-type pilot insertion, has been introduced to satisfy the need for equalizing when the channel response changes slowly within the OFDM symbol and it is required less pilot tones than the block type [6]. Then, the resultant data processed by a 2 by 2 Space Time Block Coder (STBC) developed by Alamouti [7], which is simply redistribute the data over time and space with the following codeword matrix.

$$
S=\left[\begin{array}{cc}
s_{1} & -s_{2}^{*} \\
s_{2} & s_{1}^{*}
\end{array}\right]
$$




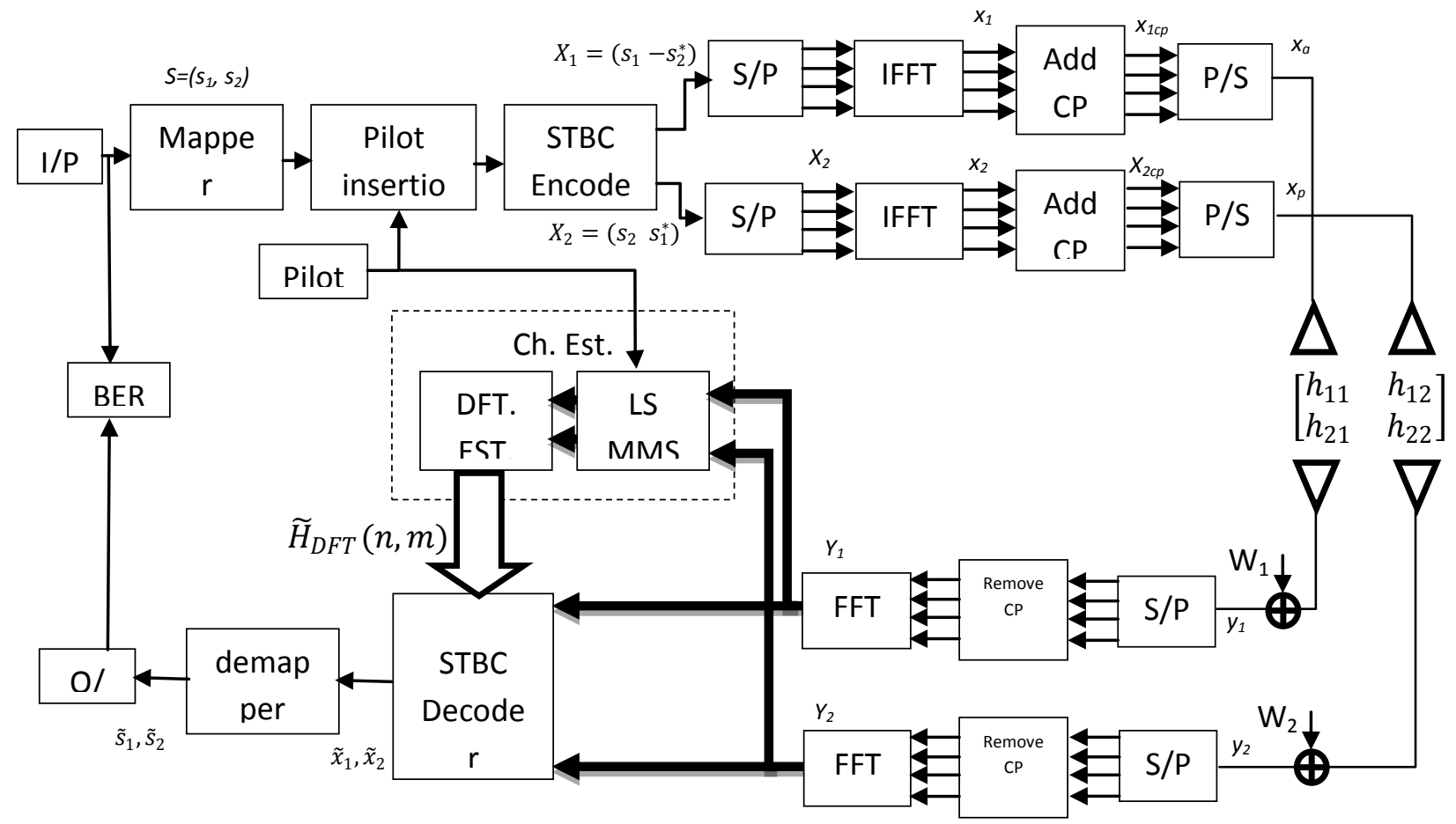

Fig 1: 2x2 MIMO-OFDM transceiver block diagram

Note that the Alamouti codeword $S$ in Equation (1) is an orthogonal matrix, i.e.

$S S^{H}=\left[\begin{array}{cc}\left|s_{1}\right|^{2}+\left|s_{2}\right|^{2} & 0 \\ 0 & \left|s_{1}\right|^{2}+\left|s_{2}\right|^{2}\end{array}\right]=\left(\left|s_{1}\right|^{2}+\left|s_{2}\right|^{2}\right) I_{2}$

Where $I_{2}$ is $2 \times 2$ identity matrix. From equation (1) the STBC process the same data over two time slot, then the transmission rate of Alamouti code is shown to be unity. And from equation (2), the key feature of STBC is that the interference between $s_{1}$ and $s_{2}$ is zero.

After the STBC, two data stream $X_{1}$ and $X_{2}$ processed by two individual OFDM modulators. Consider the first branch where:

$$
X_{1}=s_{1},-s_{2}^{*}
$$

First, $\mathrm{X}_{1}$ is converted to parallel form then N-point IFFT Npoint IFFT block used to transform the $X_{1}(\mathrm{k})$ into time domain signal $\mathrm{x}_{1}(\mathrm{n})$ as follows.

$$
\begin{aligned}
\boldsymbol{x}_{\mathbf{1}}(\boldsymbol{n}) & =\boldsymbol{I D F T}\left\{\boldsymbol{X}_{\mathbf{1}}(\boldsymbol{k})\right\} \quad \boldsymbol{n}=\mathbf{1}, \mathbf{2}, \ldots, \boldsymbol{N}-\mathbf{1} \\
& =\sum_{k=0}^{N-1} X_{1}(k) e^{j(2 \pi k n / N)}
\end{aligned}
$$

Guard interval is inserted into the OFDM symbol. The idea behind guard interval is to make the total symbol (OFDM+ guard interval) greater than or equal the maximum channel delay spread to ensure the channel effect is flat during the transmission of one OFDM symbol i.e. free ISI. Generally there are two types of guard interval, the first one is zero padding ( $\mathrm{ZP})$ that pads the guard interval with zeroes. The other way is cyclic extension of the OFDM symbol (for some continuity) to extend the OFDM symbol by copying the last samples of the OFDM symbol into its front. Let $N_{g i}$ is the length of the guard interval, and $x_{I c p}(n)$ is the resultant OFDM symbol after cyclic addition. $x_{1 c p}(n)= \begin{cases}x 1(N+n), & n=-N_{g i},-N_{g i}+1, \ldots,-1 \\ x 1(n), & n=0,1,2, \ldots, N-1\end{cases}$

(5)

Then the resultant signal converted to a serial form.

Note that same process line that applied to $X_{1}$ in the first branch is applied to $\mathrm{X}_{2}$ in the second branch to get $x_{2 c p}(n)$.

for simplicity let $x_{I c p}(n)$ and $x_{2 c p}(n)$ equal to $x_{a}$ and $x_{b}$ respectively. Then $x_{a}$ and $x_{b}$ transmitted over 2 by 2 MIMO channel $H$.

$$
H=\left[\begin{array}{ll}
h_{11} & h_{21} \\
h_{12} & h_{21}
\end{array}\right]
$$

Where, $h_{11}, h_{12}, h_{21}, h_{22}$ are the MIMO channel coefficient.

At the receiver, the received signal over the first and second antenna respectively are:

$$
\begin{aligned}
& Y_{1}=x_{a} h_{11}+x_{b} h_{21}+W_{1} \\
& Y_{2}=x_{a} h_{12}+x_{b} h_{22}+W_{2}
\end{aligned}
$$

Where $\left[W_{1}, W_{2}\right]^{\mathrm{T}}$ are AWGN for the first and second antenna respectively, $h_{m, n}$ is the channel coefficient between $m^{\text {th }}$ and $n^{\text {th }}$ antenna pair.

Again consider the first branch in the receiver in figure 1, the same process is applied to the received signal but in reversed order and also by using the pilot information the channel estimation and equalization block is applied to estimate and equalize the channel effect for each branch. At last the equalized signal is processed by STBC decoder and demapped to obtain the original data as possible. 


\section{LS AND MMSE CHANNEL ESTIMATION}

In figure 1, after inserting pilot signal along with the actual information, the resultant signal is processed by the STBC block, i.e.

$$
p_{S T B C}=\left[\begin{array}{cc}
p_{1} & -p_{2}^{*} \\
p_{2} & p_{1}^{*}
\end{array}\right]
$$

Not that since STBC process data over two time slot, the receiver has to process the data over two time slot too, then the received pilot signals over the first antenna in first and second time slot respectively are [8]

$$
\begin{aligned}
& y_{1}=p_{1} h_{11}+p_{2} h_{12}+w_{1} \\
& y_{2}=-p_{2}^{*} h_{12}+p_{1}^{*} h_{22}+w_{2}
\end{aligned}
$$

Represent equation 9 in following matrix form:

$$
\left[\begin{array}{l}
y_{1} \\
y_{2}
\end{array}\right]=\left[\begin{array}{cc}
p_{1} & p_{2} \\
-p_{2}^{*} & p_{1}^{*}
\end{array}\right]\left[\begin{array}{l}
h_{11} \\
h_{12}
\end{array}\right]+\left[\begin{array}{l}
w_{1} \\
w_{2}
\end{array}\right]
$$

Then multiplying both sides of Equation (10) by the Hermitian transpose of the pilot matrix, that is

$$
\begin{gathered}
{\left[\begin{array}{cc}
p_{1}^{*} & -p_{2} \\
p_{2}^{*} & p_{1}
\end{array}\right]\left[\begin{array}{l}
y_{1} \\
y_{2}
\end{array}\right]=\left(\left(\left|p_{1}\right|^{2}+\left|p_{2}\right|^{2}\right)\right)\left[\begin{array}{l}
h_{11} \\
h_{12}
\end{array}\right]} \\
+\left[\begin{array}{cc}
p_{1}^{*} & -p_{2} \\
p_{2}^{*} & p_{1}
\end{array}\right]\left[\begin{array}{l}
w_{1} \\
w_{2}
\end{array}\right]
\end{gathered}
$$

Then,

$$
\left[\begin{array}{l}
\hat{y}_{1} \\
\hat{y}_{2}
\end{array}\right]=\left(\left(\left|p_{1}\right|^{2}+\left|p_{2}\right|^{2}\right)\right)\left[\begin{array}{l}
h_{11} \\
h_{12}
\end{array}\right]+\left[\begin{array}{l}
\widehat{w}_{1}^{*} \\
\widehat{w}_{2}^{*}
\end{array}\right]
$$

According to [9], after ignoring the noise part in equation (11), the estimated channel $\hat{h}_{11}$ and $\hat{h}_{12}$ at pilot locations are:

$$
\begin{aligned}
& \hat{h}_{11}=\frac{y_{1} p_{1}^{*}-y_{1} p_{2}}{\left|p_{1}\right|^{2}+\left|p_{2}\right|^{2}} \\
& \hat{h}_{12}=\frac{y_{1} p_{2}^{*}+y_{1} p_{1}}{\left|p_{1}\right|^{2}+\left|p_{2}\right|^{2}}
\end{aligned}
$$

After that, the same process is applied to the second antenna to estimate $\hat{h}_{21}$ and $\hat{h}_{22}$ at pilot locations

Then the estimated MIMO channel matrix $\widehat{H}$ is

$$
\widehat{\boldsymbol{H}}=\left[\begin{array}{ll}
\hat{h}_{11} & \hat{h}_{12} \\
\hat{h}_{21} & \hat{h}_{22}
\end{array}\right]
$$

A linear interpolation is applied to estimate the channel between the pilot frequencies given by [10]

$$
\widehat{H}(k)=\widehat{H}_{m L+l}=\left(1-\frac{l}{L}\right) \widehat{H}_{m}+\left(\frac{l}{L}\right) \widehat{H}_{m+1}
$$

Where, $L$ is the number of data carrier between $m^{\text {th }}$ and $(m+l)^{t h}$ pilot, $l=1,2, \ldots . L-1, k$ is the data carrier, $m L<k<$ $(m+l) L$.

The above estimator is the $L S$ estimator, which is subject to noise enhancement in deep fading channel.

Now, consider the MMSE estimation channel in figure 2 . Using the weight matrix $W$, define $\widetilde{H}=W \widehat{H}$, which corresponds to the MMSE estimation. the Mean Square Error (MSE) of the estimated channel $\widetilde{H}$ is given as

$$
\begin{aligned}
M S E= & E\left\{|e|^{2}\right\}=E\left\{|H-W \widehat{H}|^{2}\right\} \\
& =E\left\{|H-\widetilde{H}|^{2}\right\}
\end{aligned}
$$

Where,

$$
W=R_{H \widehat{H}} R_{H \widehat{H}}^{-1}
$$

Where $R_{H H}$ is the cross-correlation between the true channel vector and LS estimated channel vector, $R_{\hat{H} H}$ is the autocorrelation matrix of $\widehat{H}$. Then MMSE channel estimation defined as

$$
\widetilde{H}=\left(R_{H \hat{H}} R_{H H}^{-1}\right) \widehat{H}
$$

Fig 2: MMSE channel estimation

Its obvious that MMSE channel estimation method shows better estimate in terms of W in such a way that the MSE in equation (15) is minimized [11].

\section{ESTIMATION ENHANCED BASED ON DFT INTERPOLATION}

Figure 3 illiterate the concept of DFT channel estimation technique that used to improve the performance of LS or MMSE channel estimators by reducing the noise effect outside the maximum channel delay. First the frequency response of the noisy estimated channel is transformed to the time domain by an IDFT operation, and then transformed back to the frequency domain by a DFT after appropriately being processed in order to reduce noise effects [12]. DFT channel estimation with comb-type pilot proceeds as following [13]:

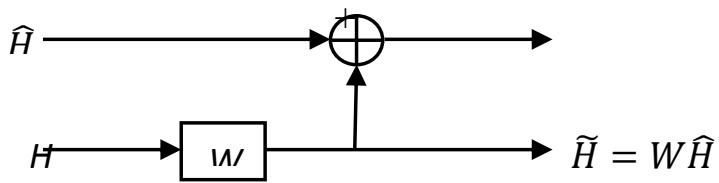

Fig 3: DFT-based channel estimation

Step1: Let $\widetilde{H}(k)$ represent the estimated channel gain at the $k^{\text {th }}$ subcarrier, which obtained by $L S$ and/or $M M S E$ channel estimator. Taking the IDFT operation to the estimated channel.

$$
h(n)=\operatorname{IDFT}\{\widetilde{H}(k)\}=\sum_{k=0}^{N-1} \widetilde{H}(k) e^{j\left(\frac{2 \pi k n}{N}\right)}
$$

Step2: In time domain the following processing is applied to reduce the noise effect .The most channel power concentrates on only small parts of the estimated time-domain samples that are transformed by IDFT (called the significant taps) .A straight forward way is to ignore (zero padding) the coefficients, called as the non-significant taps, in which they are very noisy compared to the significant taps. Then

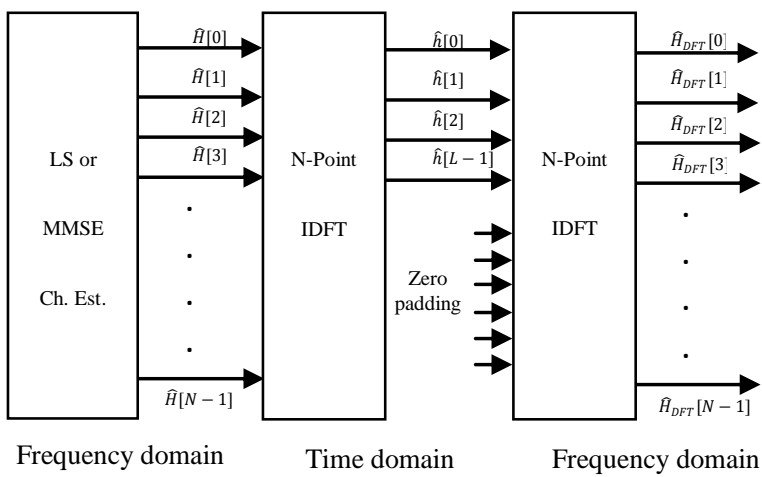

transforming only the remaining coefficients. 


$$
h(n)=\left\{\begin{array}{l}
h(n) \quad, n=0,1, \ldots, \beta \\
0 \quad, n=\beta+1 \ldots, N-1
\end{array}\right.
$$

Usually the window length $\beta \leq L$ can be set as the sampled maximum excess delay of the channel.

Step3: take N-point DFT of the estimated channel impulse response after ignoring the non-significant taps to obtain the estimated channel frequency response of length-N

$$
=\frac{1}{N} \sum_{k=0}^{N-1} h(n) e^{-j\left(\frac{2 \pi k n}{N}\right)}
$$

Represent $\widetilde{H}_{D F T}(\mathrm{k})$ in matrix form i.e.

$$
\widetilde{H}_{D F T}=\left[\begin{array}{ll}
\widetilde{H}_{11_{D F T}} & \widetilde{H}_{12_{D F T}} \\
\widetilde{H}_{21_{D F T}} & \widetilde{H}_{22_{D F T}}
\end{array}\right]
$$

Finally after the channel estimator, the equalized signal after STBC decoder given as:

$$
\begin{aligned}
& \tilde{x}_{1}=\widetilde{H}_{11_{D F T}}^{*} y_{1}+\widetilde{H}_{12_{D F T}} y_{2}^{*} \\
& \tilde{x}_{2}=\widetilde{H}_{21_{D F T}}^{*} y_{1}-\widetilde{H}_{22_{D F T}} y_{2}^{*}
\end{aligned}
$$

\section{SIMULATION RESULTS}

The setting parameter for MIMO-OFDM system used in the simulation are indicated in table 1 .

Table 1. Simulation parameter

\begin{tabular}{|c|c|}
\hline Parameter & Value \\
\hline FFT size (total carriers) & 64 \\
\hline Guard interval & 16 (25\% FFT size) \\
\hline Guard type & Cyclic extension \\
\hline Pilot carriers & 16 \\
\hline Signal constellation & BPSK \\
\hline Number of transmit antenna & 2 \\
\hline Number of receive antenna & $\begin{array}{c}\text { (LS \& MMSE) with and } \\
\text { without DFT }\end{array}$ \\
\hline Channel estimator &
\end{tabular}

First we run the simulation for the OFDM system only and applying the LS \& MMSE estimators both with and without DTF estimation. It is obvious in figure 4 that LS (red line) requires about $(5-8) \mathrm{dB}$ higher than MMSE (blue line) to achieve the same BER $\left(10^{-3}\right)$. This is because the LS estimator is subject to noise enhancement especially when the channel in deep fading. The performance of estimation can be improved by increasing the number of pilots. But on the other hand, increasing pilot tone reduces data transmission efficiency. By using DFT estimation on both LS and MMSE without increasing the number if pilot data, we can see that there is a noticeable improvement on LS (purple line) and MMSE (green line) by $5 \mathrm{~dB}$ and (1-1.5) dB respectively.

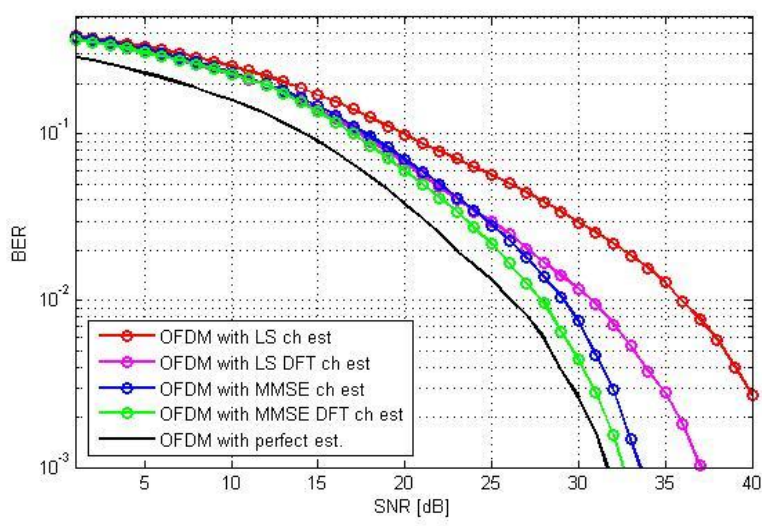

Fig 4: BER performance of OFDM system witd LS and MMSE both with and without DFT estimation technique

The second simulation run is combining the MIMO antenna configuration with the OFDM system, and by taking the advantage of diversity technique we can see that the system performance is remarkably improved as shown in figure 5 .

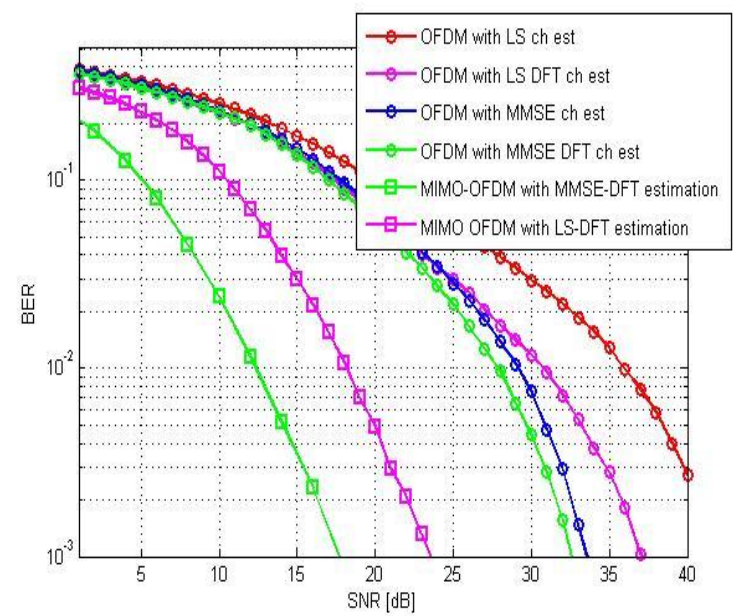

Fig 5: BER performance of MIMO-OFDM system with LS and MMSE both with DFT estimation technique

\section{CONCLUSION}

In this paper, a new channel estimation method has been proposed to enhance the performance of the most popular channel estimators (LS d MMSE) based on comp type pilot arrangement in MIMO-OFDM system. MMSE estimator shows butter performance than LS estimator due to its ability to minimize the MSE using the weight matrix W, farther improvement on LS and MMSE estimators using DFT-based estimation technique due to the ability of this method to reduce the noise effect outside the maximum channel delay. Therefore combining DFT channel estimation with LS and MMSE helps the estimate the channel ac correct as possible to improve the performance of the MIMO-OFDM system which became a promising technology for wireless communication systems such IEEE $802.11 \mathrm{a} / \mathrm{g} / \mathrm{n}$, WiMAX and LTE, 


\section{ACKNOWLEDGMENTS}

Our thanks to the experts who have contributed towards development of this paper

\section{REFERENCES}

[1] Rashmi Mishra, Baibaswata Mohapatra "PERFORMANCE EVALUATION OF OFDM SYSTEM" International Journal of Engineering and Advanced Technology (IJEAT), ISSN: 2249 - 8958, Volume-1, Issue-3, February $2012 \mathrm{~F}$

[2] Lakshmi Prasanna.P, G.Rama Krishna , "DIVERSITY analysis in MIMO systems using OSTBC(Orthogonal Space Time Block Codes," International Journal of Emerging Technology and Advanced Engineering, ISSN 2250-2459, Volume 2, Issue 9, September 2012

[3] Angel Lozano, Senior Member, IEEE, and Nihar Jindal, "Transmit Diversity vs. Spatial Multiplexing in Modern MIMO Systems," IEEE TRANSACTIONS ON WIRELESS COMMUNICATIONS, VOL. 9, NO. 1, JANUARY 2010F

[4] Quan Zhou, Hongyuan Zhang, Huaiyu Dai, “Adaptive Spatial Multiplexing Techniques for Distributed MIMO Systems," _2 2004 Conference on Information Sciences and Systems. The Princeton University. March 17-19. 2004F

[5] Marcello Cicerone, Osvaldo Simeone, Umberto Spagnolini, "Channel Estimation for MIMO-OFDM Systems by Modal Analysis/Filtering," IEEE TRANSACTIONS ON COMMUNICATIONS, VOL. 54, NO. 11, NOVEMBER 2006F

[6] Lilong Liu, Xuelin Yang, Jun Li ; Meihua Bi, Hao He, Weisheng $\mathrm{Hu}$, "Experimental evaluation of pilot arrangement for channel estimation in OFDM systems," IEEE Communications and Photonics Conference and Exhibition, 2011. ACP. Asia, Page(s): $1-6 \mathrm{~F}$

[7] Alamounti, S.M. (1998) A simple transmit diversity scheme for wireless communications. IEEE J. Select. Areas Commun., 16(8), 1451-1458

[8] S. M. Alamouti, "A simple transmit diversity technique for wireless communications," IEEE Journal on Selected Areas in Communications, vol. 16, pp. 1451-1458, 1998.

[9] F.Delestre, Y.Sun, "Pilot Aided Channel Estimation for MIMO-OFDM Systems," London Communications Symposium 2009H

[10] Kala Praveen Bagadi, Susmita Das. "MIMO-OFDM Channel Estimation using Pilot Carries," International Journal of Computer Applications (0975 - 8887) Volume 2 - No.3, May 2010 G

[11] Yong Soo Cho, Jaekwon Kim, Won Young Yang, Chung G. Kang . (2010). MIMO-OFDM Wireless Communications with MATLAB. Asia: John Wiley \& Sons Pte Ltd.

[12] ] Xu Yan , Lin Ying, "Research of OFDM systems channel estimation based on DFT improved algorithm," IEEE 4th International Conference on Computer Science \& Education, 2009. ICCSE '09. Page(s) 250-253.

[13] Mahdi Abdul Hadi, Omar Almukhtar Tawfeeq. "Channel Estimation Enhancement in OFDM System based on Time Domain interpolation," International Journal of Computer Applications (0975 - 8887) Volume 97No.11, 2014 